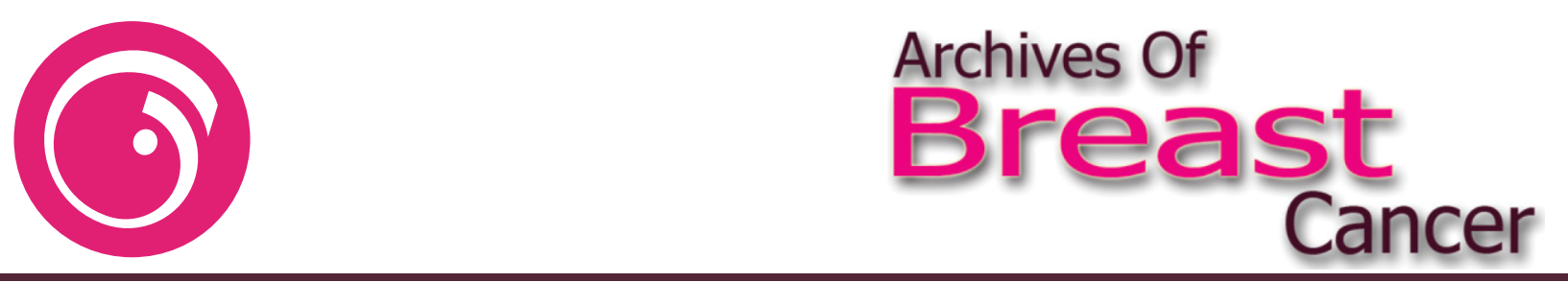

DOI: $10.32768 / a b c .20218144-50$

\title{
The Association of Neoadjuvant Systemic Treatment on Hormone Receptor and Her2 Expression in Breast Cancer
}

\author{
William MacFaul $^{\star a}$, T Michael D Hughesa ${ }^{\mathrm{a}, \mathrm{b}, \mathrm{c}}$, Kerry Hitosd ${ }^{\mathrm{d}, \mathrm{e}}$, Nirmala Pathmanathane ${ }^{\mathrm{f}, \mathrm{g}}$, \\ Nicholas K Nguiaa,b,c \\ ${ }^{a}$ The University of Sydney, Sydney Adventist Hospital Clinical School, Sydney, NSW, Australia \\ ${ }^{b}$ Breast Multidisciplinary Team, Sydney Adventist Hospital, Sydney, NSW, Australia \\ ${ }^{\circ}$ Division of Surgery, Sydney Adventist Hospital, Sydney, NSW, Australia \\ ${ }^{d}$ Westmead Research Centre for Evaluation of Surgical Outcomes, Department of Surgery, Westmead \\ Hospital, Sydney, NSW, Australia \\ ${ }^{e}$ Westmead Clinical School, The University of Sydney, NSW, Australia \\ ${ }^{f}$ Westmead Breast Cancer Institute, Westmead, NSW, Australia \\ ${ }^{g}$ Douglass Hanly Moir Pathology, Macquarie Park, NSW, Australia
}

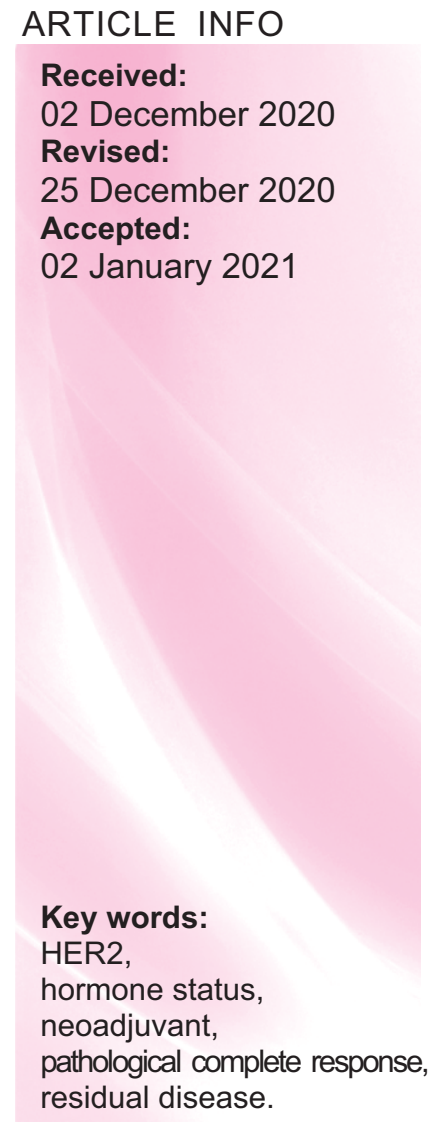

ARTICLE INFO

Received:

02 December 2020

Revised:

Accepted:

02 January 2021

Key words:

hormone status

pathological complete response, residual disease.

\section{ABSTRACT}

Background: Pathological complete response (pCR) following neoadjuvant systemic treatment(NAST) for breast cancer is associated with improved prognosis; however, a large proportion of patients have residual disease. Oestrogen Receptor (ER) and HER2 status have been shown to affect likelihood of achieving $\mathrm{pCR}$, with ER positive tumors being more treatment resistant. As hormone receptor status is heterogeneous within tumors, we postulated that, following NAST, ER expression would change in patients with residual disease, as the ER negative cells within the tumor are more treatment sensitive.

Methods: A retrospective case series of patients treated with NAST prior to surgery at our institution was conducted. Information collected included demographic data, tumor grade, hormone receptor and HER2 status both before and after treatment, and pCR rates.

Results: Of the 44 patients included, half achieved pCR. HER2 status $(\mathrm{P}=0.01)$, and subtype $(\mathrm{P}=0.008)$ were significantly associated with pCR. HER2 positive/ER negative tumors were most likely to undergo pCR. Approximately $80 \%$ of residual disease was ER positive. Higher levels of ER expression were also associated with increasing residual cancer burden $(\mathrm{RCB})$ class $(\mathrm{P}=0.037)$. There was no trend between change in ER or HER2 expression following NAST. Median change in ER expression was $80 \%$ to $90 \%(\mathrm{P}=0.89)$, HER2 intensity changed from 3.0 to $2.2(\mathrm{P}=0.67)$ following treatment.

Conclusion: Consistent with the literature, we have shown associations between ER and HER2 status and PCR, and between ER expression and residual disease burden. Our study was not able to demonstrate a significant trend in hormone and HER2 expression.

\footnotetext{
*Address for correspondence:

William MacFaul, MB ChB

Address: SAN Clinic, Suite 404, Sydney Adventist Hospital, Sydney, New South Wales 2071, Australia.

Tel: +61294879111

Email:willmacfaul@gmail.com
}

\section{Introduction}

Breast cancer is the second leading cause of death from cancer in women worldwide, with around 18,000 Australians diagnosed with breast cancer each year. ${ }^{1,2}$ Over recent years neoadjuvant systemic therapy (NAST), which entails systemic therapy prior to definitive surgery, has been widely used with 
the aim of downstaging the breast cancer and deescalating the extent of breast and axillary surgery. ${ }^{3}$

Pathological complete response ( $\mathrm{pCR}$ ), defined as absence of residual invasive disease in the breast and in lymph nodes following NAST, has been proposed as a surrogate marker for treatment efficacy, as pCR has been shown to confer both an improved disease-free and overall survival. ${ }^{4} \mathrm{~A}$ pooled analysis of 12 international neoadjuvant therapy trials, found that $\mathrm{pCR}$ was associated with increased overall survival, and that patients with ER positive, HER 2 negative cancers were least likely to achieve pCR, while triple negative and HER2 positive tumors had the highest pCR rates. ${ }^{4}$

Other trials have further demonstrated the significance of hormone and HER 2 status on pCR. A multi-centre study found that $\mathrm{pCR}$ rates were lower in ER positive cancer subtypes, luminal A and B cancers. ${ }^{5}$ Carey et al. assessed response to treatment depending on genomic subtype and found that response to treatment was approximately double in HER2 enriched rather than Luminal A and B subtypes. ${ }^{6}$ This study also looked at the residual disease tumor biology following treatment and found lower HER2 enriched cell types in those with residual disease; there was also a shift in intrinsic subtype within tumors which were treatment resistant. ${ }^{6}$ They noted shifts towards Luminal A subtypes from Luminal B and HER2 enriched tumors following treatment. This may also reflect tumor heterogeneity with neoadjuvant treatment eliminating the chemo-sensitive cell population but having little or no effect on the chemo-resistant cell population. ${ }^{6}$ However, it may also suggest some alteration of tumor biology from the NAST itself.

These studies highlight the significance of the heterogeneity within breast cancers and how this impacts response to treatment and prognosis. ${ }^{4-6}$ These trials demonstrate that ER negative and HER2 positive cancers are more likely to achieve $\mathrm{pCR} .^{4-7}$ Furthermore, given the overall pCR rates in these studies were $56 \%, 46 \%$, and $18 \%$, this leaves a significant proportion of patients with residual disease found at surgery. ${ }^{4-6}$

Further understanding of the biology of residual disease has important treatment implications. Findings from the multicentre open label, phase II randomised NeoSphere trial evaluated the efficacy of dual anti-HER2 therapy. Results from this study showed that even in patients who did not undergo a $\mathrm{pCR}$, there was a progression free survival benefit in those treated with dual anti-HER 2 therapy combined with chemotherapy compared with single agent therapy. ${ }^{8}$ The CREATE-X trial investigated the treatment benefit of ongoing adjuvant capecitabine for patients with residual disease following NAST in HER2 negative patients. ${ }^{9}$ They randomised patients with residual disease to receive either a further 8 cycles of capecitabine or no further treatment and found an increased overall survival and disease free survival in the treatment arm with the greatest benefit in the triple negative cohort. ${ }^{9}$ To ensure that these findings were not related simply to length of systemic treatment, further studies have given additional chemotherapy to $\mathrm{pCR}$ patients and have not demonstrated any further benefit, suggesting that the additional treatment only seems to benefit those with residual disease..$^{10}$

Evidence from neoadjuvant trials shows pCR is an important determinant of disease prognosis in breast cancer and that in those patients who have failed to undergo $\mathrm{pCR}$, there is a reduced duration of survival. ER negative cancers and HER2 positive cancers are much more sensitive to NAST and more likely to undergo $\mathrm{pCR}$.

We postulate that in ER positive cancer the population of cells which have nil or lower oestrogen receptor expression are more responsive to systemic chemotherapy than cells which have a high level of oestrogen receptor expression. If this theory were true, then one would expect that the percentage of ER receptor expressing cells would be lower in the pre-operative biopsy compared to the post NAST surgical specimen. Similarly, the HER2 status may also show changes following NAST- possibly explaining the subtype changes that have been seen in previous studies.

The primary aim of this study was to evaluate whether there is a change in hormonal and HER2 receptor expression between the pre-neoadjuvant core biopsies compared with residual disease following NAST, with a secondary aim of evaluating local pCR rates across subtypes.

\section{Methods}

We conducted a retrospective study of patients treated at the Sydney Adventist Hospital, Sydney, Australia over a 10-year period from 2009 to 2019. The breast cancer database was searched to identify patients who had undergone NAST followed by definitive surgery. The medical records of these patients were reviewed to retrieve clinical details and pathological information.

The inclusion criteria included early and locally advanced breast cancer, treatment with neoadjuvant therapy prior to definitive surgical management. Pre-operative data included patient demographics, histological evidence of lymph node involvement, tumor type and grade, hormone (ER and PR) status (percentage and intensity), and HER2 status by immunohistochemistry or in situ hybridisation (SISH or FISH).

ER and PR status were defined as positive if there were at least $1 \%$ positive tumor nuclei stained as per The American Society of Clinical Oncology and the College of American Pathologists Guidelines which classifies Oestrogen receptor as weakly positives if it is between $1-10 \%$. 
Type and length of NAST were recorded as was the type of procedure on the breast and axilla. The operative pathology report was examined for presence of residual disease or PCR, tumor grade, ER and PR status with percentage and intensity, HER2 status, lymph node status with the number of positive nodes, and Residual Cancer Burden (RCB) class. pCR rates were defined as no residual invasive cancer in the breast and axillary lymph node(s), including isolated tumor cell in the lymph node after surgery, in keeping with the current 8th edition of the AJCC cancer staging manual. ${ }^{12}$

The residual cancer burden ( $\mathrm{RCB})$ index is a standardised calculated score based on pathological data which is usually provided within the pathology report. ${ }^{13}$ Five variables are included in the calculation, namely, primary tumor bed area $(\mathrm{mm} \mathrm{x}$ $\mathrm{mm}$ ), percentage overall cancer cellularity, percentage of cancer that is in-situ disease, number of positive lymph nodes, and diameter of largest metastasis.

This study was granted approval by Adventist Healthcare Limited Human Research Ethics Committee, in April 2019, and was carried out in accordance with the National Health and Medical Research Council, Medical National Statement on Ethical Conduct in Human Research. Patient consent for research was obtained as part of the consent for treatment.

For the statistical analysis, matched pairs were compared for percentage and intensity across ER, PR and HER2. Chi squared test for trends, or ManWhitney U test was used to analyse differences in ER expression and HER2 intensity. Subgroups analysed for difference in pCR rates were ER positive/HER negative low grade, ER positive/ HER negative high grade, ER positive/HER positive and triple negative. Low grade incorporated Grade 1 and 2 tumors, high grade referring to Grade 3 tumors. This is similar to grouping conducted by a previous large multicentre pooled analysis. ${ }^{4}$ For non-parametric data distribution, the medians and where possible the interquartile range was reported. The interquartile range was defined as the $25^{\text {th }}$ to $75^{\text {th }}$ percentile (IQR), parametric continuous data were reported with the mean, range and where possible the $95 \%$ confidence intervals were included. All tests were two tailed and statistical significance was set at $\mathrm{P}<0.05$. All analyses were performed using IMB SPSS Statistics for Windows, Version 26 (IBM SPSS Statistics for Windows, IBM Corporation, Armonk, NY).

\section{Results}

In total, 62 female patients treated over a 10-year period were identified as suitable from the breast cancer database. Forty-four women met the inclusion criteria and had complete data for analysis. Baseline clinicopathological data is summarised in Table 1.
NAST consisted of the following: 20 patients $(45 \%)$ received Pertuzumab, Trastuzumab, and Taxane (Paclitaxel), the remainder received differing combinations of chemotherapy, the most common being doxorubicin hydrochloride (Adriamycin), cyclophosphamide, and paclitaxel (AC-T treatment).

In total, six patients (14\%) did not complete the full neoadjuvant treatment due to a range of different side effects.

Following NAST, 27 patients (63\%) underwent wide local excision as definitive surgical treatment, with the remainder undergoing mastectomy (47\%). In terms of axillary management, $26(60 \%)$ of patients had a sentinel lymph node biopsy, 16 (37\%) patients had a full axillary dissection, with one patient having no axillary management. Overall, 12 patients $(27 \%)$ were lymph node positive following treatment.

Table 1. Clinicopathological Data

\begin{tabular}{lcc}
\hline Pre-Operative Data & & Frequency (\%) \\
\hline Age (years) & & Median (IQR) \\
Tumor Type & & $54(49-64)$ \\
& Ductal & $39(88.6)$ \\
& Lobular & $4(9.1)$ \\
Node status & Other & $1(2.3)$ \\
& Node positive & $18(40.9)$ \\
& Node negative & $26(59.1)$ \\
Grade & 1 & $19(43.2)$ \\
& 2 & $22(50)$ \\
Oestrogen Status & 3 & $3(6.8)$ \\
& ER -ve & $15(34.1)$ \\
Progesterone Status & ER +ve & $29(65.9)$ \\
& PR +ve & $24(43.2)$ \\
HER2 Status & PR -ve & $20(54.5)$ \\
& HER2 -ve & $14(31.8)$ \\
& HER 2 +ve & $30(68.2)$ \\
\hline
\end{tabular}

\section{Residual Disease}

Twenty-two patients had residual disease, ER pos/HER2 neg cancers made up $54.6 \%$ of residual disease subtype, with $45.5 \%$ being ER pos low grade, and $9.1 \%$ being ER pos high grade. HER2 pos/ER pos cancers made up $31.8 \%$ of patients with residual disease, with triple negative cancers and HER2 pos/ER neg cancers making up 9.1 and triple negative cancers $4.5 \%$ of residual disease.

For the 29 patients with ER positive cancer, the median percentage of oestrogen positive cells prior to treatment was $80 \%$ (IQR 1-90), and the median percentage following treatment was $90 \%$ (IQR 5095) (p-value across both medians 0.89).

Treatment was associated with a decrease in positivity in seven of 15 ER positive tumors with residual disease, with treatment associated with an increase in $4 / 15$, and no change in $3 / 15$. One patient's ER expression was associated with a change from 

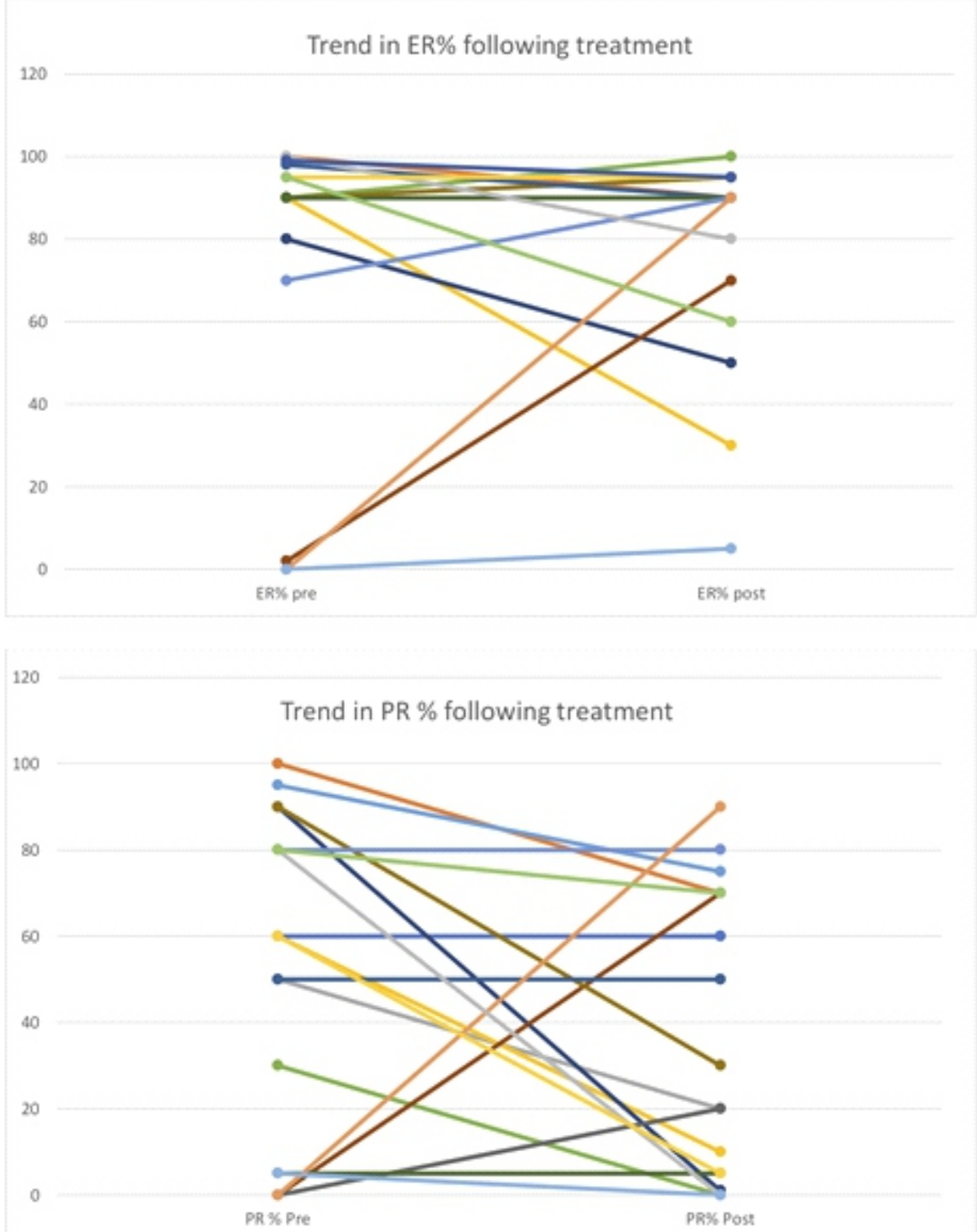

Figure 1. Map of each individual patient ER \& PR percentage and HER2 intensity between baseline and after neoadjuvant therapy

$0 \%$ ER expression (ER negative), to $5 \%$ ER expression (ER positive) following neoadjuvant treatment. The median percentage of progesterone expression was $45 \%$ (IQR: $0.25-75$ ) pre-treatment and $30 \%$ following treatment (IQR $0.00-70$, $\mathrm{P}=0.77)$. HER 2 intensity pre-treatment was 3.0 (IQR 2-3) and 2.2 (IQR 1-3) after treatment $(\mathrm{P}=0.67)$. The difference in expression for individual patient associated with treatment for oestrogen and progesterone percentage, and HER 2 intensity can be seen in Figure 1. None of these associated changes reached statistical significance.

The range of difference of ER percentage in tumor cells of patients following treatment compared to diagnostic biopsy was between -35 to +90 , and the change in PR expression was between 85 to +75 .

RCB class was available for 15 out of $22(68 \%)$ of patients with residual disease. RCB class was associated with oestrogen positivity; the higher the ER percentage prior to treatment, the higher the RCB class $(\mathrm{P}=0.037)$.

\section{Pathological Complete Response}

Following treatment, $22(50 \%)$ patients had a pCR, leaving $22(50 \%)$ with residual disease. When pCR rates were evaluated by hormone status, ER negative cancers were three times more likely to undergo pCR than ER positive cancers (OR 2.97; $95 \%$ CI $0.8-10.8 ; \mathrm{P}=0.06$ ).

HER2 positive patients were more likely to undergo pCR than HER 2 negative, with 19 out of 30 HER2 positive patients achieving pCR compared to 3 out of 14 HER 2 negative $(\mathrm{P}=0.01)$. Patients were six times more likely to undergo pCR if they were HER2 positive than HER2 negative (OR 6.33; 95\% CI $1.4-27 ; \mathrm{P}=0.01)$.

When subtype was evaluated by $\mathrm{pCR}$, the 
patients most likely to achieve pCR were HER2 pos/ER neg patients, with $80 \%$ of these achieving pCR, followed by HER pos/ER pos with 55\% and triple negative, with $50 \%$ achieving pCR $(\mathrm{P}=0.008)$. No HER2 neg/ER pos cancers $(n=8)$ in our study underwent pCR.

\section{Discussion}

In this retrospective study we found that of the $50 \%$ of our patients with residual disease, patients were more likely to be ER positive prior to treatment, but this was just short of statistical significance $(\mathrm{P}=$ $0.06)$. When residual disease was broken down by subtype, ER pos/Her neg were the most common, but interestingly despite a high pCR rate of $56 \%$ for HER2 pos/ER pos patients, a large proportion of patients with residual disease were HER2 pos/ER pos (31.8\%). Overall, of those with residual disease, $86 \%$ were made up of ER positive cancers, highlighting the impact of hormone status on residual disease biology. The low proportion of triple negative cancers with residual disease is a positive finding given the generally poor prognosis of these tumors.

There was no apparent statistically significant treatment effect on oestrogen or progesterone percentage or intensity $(\mathrm{P}=: 0.82, \mathrm{P}=0.86)$. HER2 expression was significantly associated with $\mathrm{pCR}$ $(\mathrm{P}=0.01)$.

Despite the associated differences in individual patients' ER and PR expression in the tumor following neoadjuvant treatment, there was no overall pattern observed in the direction or magnitude of change.

The main aim of this study was to assess the impact of NAST on hormone receptor expression in breast cancer cells. A change in hormone receptor expression has been reported previously in the literature. Van de Ven et al. found that of 10 trials that assessed for change in ER expression levels, 4 trials, including a large retrospective study, found a changes in ER expression, with both increases and decreases being observed, as found in this study. ${ }^{14-17}$

Our failure to detect a significant change within ER expression could be related to the high ER expression of $>90 \%$ on initial biopsy in over $50 \%$ of ER positive patients. Our hypothesis that the ER percentage may increase would be difficult to detect in these patients due to such a small proportion of ER negative cells.

There are two outliers that are worthy of discussion. One patient's ER expression was $1-2 \%$ on diagnostic biopsy and $90 \%$ following NAST. On further examination, it appeared that the patient had a number of possible satellite lesions in addition to the lesion that was biopsied. It may be that this discordance could be explained by a pCR in the original lesion, and the different hormone profile on the surgical histology is from concurrent cancer.
Similarly, a difference in receptor expression across treatment from $2 \%$ to $70 \%$ following NAST in a different patient appears to be related to a $\mathrm{pCR}$ in the breast from the original lesion, with the hormone profile taken from a metastatic deposit within the lymph node. Such outliers may have affected our results given the small number of patients in the study; furthermore, some hormone negative and HER2 negative tumors became positive due to tumor heterogeneity which the small core biopsy sample may not detect.

An interesting finding in our study was that ER percentage prior to treatment was the relationship between ER positivity and RCB class. RCB class has been shown to be prognostic for long term survival, and therefore has been proposed as a surrogacy for treatment efficacy in those patients who do not undergo $\mathrm{pCR}$. The association between an increased ER expression and increased RCB class may be related to the fact that ER positivity is known to impact pCR. However, it is interesting as ER status in regards to pCR was not found to be statistically significant in this study, whilst association with RCB class was. This would suggest that not only does oestrogen positivity predict likelihood of having residual disease, but also the extent of the residual disease. It may also be worth considering comparing scores that combine intensity and expression, such as the Allred score or $\mathrm{H}$ score, before and after NAST to see whether combining these factors allows more of a treatment effect to be identified.

The pCR rate in our population of $50 \%$ is comparable to other landmark studies. The TRYPHAENA trial, which assessed pCR rates across over 200 patients receiving anti-HER2 therapy in combination with chemotherapy, reported pCR rates of 56 and $55 \%$ for the two arms of the trial. The NeoSphere trial, which similarly compared differing anti-HER 2 regimens, reported $\mathrm{pCR}$ rates of $46 \%$ for the combined HER2 therapy arm. ${ }^{18,19}$ The GBG GeparSepto study comparing anti HER2 chemotherapy across over 1000 patients with early breast cancer reported a pCR rate of $56 \%{ }^{20}$ Our high pCR rate did limit the number of patients with residual disease, which was unfortunate given that our primary objective was the impact on residual disease.

When our pCR rates were broken down by hormone status, patients were more likely to undergo pCR if they were ER negative, but this was just short of clinical significance ( $\mathrm{p}$-value 0.06 ). This finding is in line with evidence in the literature that ER negative patients are more likely to undergo $\mathrm{pCR}^{21}$. Our finding that HER2 positive patients were significantly more likely to undergo $\mathrm{pCR}$, is also in line with the literature.

It appears that there may be interplay between HER2 and ER signalling pathways and this 
interaction may impact response to treatment. ${ }^{22}$ The 'Cross-talk' hypothesis suggests that oestrogen binding to cytoplasmic oestrogen receptors in breast cancer cells activates signalling pathways that bypass the blockage of HER2 by anti-HER2 chemotherapy agents which may therefore lead to increased resistance to anti-HER2 treatment in HER 2 pos/ER pos cancers. ${ }^{22,23}$ PCR rates have even been shown to differ within HER2 pos/ER pos cancers with an inverse relationship between pCR rate and ER percentage. ${ }^{24}$ Due to the small sample size in this study, we could not explore this relationship.

This retrospective, single institution study investigating the effect of NAST on early breast cancer produced a number of important findings which we feel add to the current literature. Whilst there have been many studies on pCR, there have been few on residual disease; our study provides further information on the biology of residual disease. Over $80 \%$ of patients with residual disease were ER positive. Furthermore, increasing ER percentage in the pre-treatment biopsy was associated with increasing residual cancer burden following treatment, providing implications of hormone status on both likelihood and volume of residual disease.

The study confirms that ER positive tumors are less likely to have $\mathrm{pCR}$, an important observation to acknowledge when advising patients of likely outcomes from NAST.

Additionally, our study provides further information to the literature on $\mathrm{pCR}$. We demonstrated an overall pCR rate of $50 \%$, with HER2 positive and ER negative patients much more likely to undergo pCR. Notably, when these two characteristics were combined, $80 \%$ of HER2 positive/ER negative patients achieved pCR. The study was limited by the small numbers, but in spite of this it has shown that ER status and HER2 are not significantly impacted by NAST when there is residual disease to assess.

\section{Conflicts of Interest}

There are no conflicts of interest to declare, and there was no external funding for the study.

\section{References}

1. Comprehensive molecular portraits of human breast tumors. Nature. 2012;490(7418):61-70.

2. Siegel RL, Miller KD, Jemal A. Cancer statistics, 2019. CA Cancer J Clin. 2019;69(1):7-34.

3. Killelea BK, Yang VQ, Mougalian S, Horowitz NR, Pusztai L, et al. Neoadjuvant chemotherapy for breast cancer increases the rate of breast conservation: results from the National Cancer Database. J Am Coll Surg. 2015;220(6):1063-9.

4. Cortazar P, Zhang L, Untch M, Mehta K, Costantino JP, et al. Pathological complete response and long-term clinical benefit in breast cancer: the CTNeoBC pooled analysis. Lancet. 2014;384(9938):164-72.

5. Gavila J, Oliveira M, Pascual T, Perez-Garcia J, Gonzalez X, et al. Safety, activity, and molecular heterogeneity following neoadjuvant nonpegylated liposomal doxorubicin, paclitaxel, trastuzumab, and pertuzumab in HER2-positive breast cancer (Opti-HER HEART): an openlabel, single-group, multicenter, phase 2 trial. BMC Med. 2019;17(1):8.

6. Carey LA, Berry DA, Cirrincione CT, Barry WT, Pitcher BN, et al. Molecular Heterogeneity and Response to Neoadjuvant Human Epidermal Growth Factor Receptor 2 Targeting in CALGB 40601, a Randomized Phase III Trial of Paclitaxel Plus Trastuzumab With or Without Lapatinib. J Clin Oncol. 2016;34(6):542-9.

7. Jones RL, Salter J, A'Hern R, Nerurkar A, Parton $\mathrm{M}$, et al. Relationship between oestrogen receptor status and proliferation in predicting response and long-term outcome to neoadjuvant chemotherapy for breast cancer. Breast Cancer Res Treat. 2010;119(2):315-23.

8. Gianni L, Pienkowski T, Im YH, Tseng LM, Liu $\mathrm{MC}$, et al. 5-year analysis of neoadjuvant pertuzumab and trastuzumab in patients with locally advanced, inflammatory, or early-stage HER2-positive breast cancer (NeoSphere): a multicentre, open-label, phase 2 randomised trial. Lancet Oncol. 2016;17(6):791-800.

9. Zujewski JA, Rubinstein L. CREATE-X a role for capecitabine in early-stage breast cancer: an analysis of available data. NPJ Breast Cancer. 2017;3:27.

10. Gluz O, Nitz U, Liedtke $C$ et al. No survival benefit of chemotherapy escalation in patients with $\mathrm{pCR}$ and high-immune triple-negative early breast cancer in the neoadjuvant WSGADAPT-TN trial. San Antonio Breast Cancer Symposium 2018; Abstr. GS5-06.

11. Hammond ME, Hayes DF, Dowsett M, Allred DC, Hagerty KL, et al. American Society of Clinical Oncology/College Of American Pathologists guideline recommendations for immunohistochemical testing of estrogen and progesterone receptors in breast cancer. J Clin Oncol. 2010;28(16):2784-95.

12. Amin MB ES, Greene F, Byrd DR, Brookland RK, Washington MK, Gershenwald JE, Compton CC, Hess KR, et al. AJCC Cancer Staging Manual (8th edition): Springer International Publishing: American Joint Commission on Cancer; 2017.

13. Symmans WF, Peintinger F, Hatzis C, Rajan R, Kuerer H, et al. Measurement of residual breast cancer burden to predict survival after neoadjuvant chemotherapy. J Clin Oncol. 2007;25(28):4414-22. 
14. van de Ven S, Smit VT, Dekker TJ, Nortier JW, Kroep JR. Discordances in ER, PR and HER2 receptors after neoadjuvant chemotherapy in breast cancer. Cancer Treat Rev. 2011;37(6):422-30.

15. Colleoni M, Viale G, Zahrieh D, Pruneri G, Gentilini O, et al. Chemotherapy is more effective in patients with breast cancer not expressing steroid hormone receptors: a study of preoperative treatment. Clin Cancer Res. 2004;10(19):6622-8.

16. Piper GL, Patel NA, Patel JA, Malay MB, Julian TB. Neoadjuvant chemotherapy for locally advanced breast cancer results in alterations in preoperative tumor marker status. Am Surg. 2004;70(12):1103-6.

17. Makris A, Powles TJ, Allred DC, Ashley SE, Trott PA, et al. Quantitative changes in cytological molecular markers during primary medical treatment of breast cancer: a pilot study. Breast Cancer Res Treat. 1999;53(1):51-9.

18. Gianni L, Pienkowski T, Im YH, Roman L, Tseng LM, et al. Efficacy and safety of neoadjuvant pertuzumab and trastuzumab in women with locally advanced, inflammatory, or early HER2positive breast cancer (NeoSphere): a randomised multicentre, open-label, phase 2 trial. Lancet Oncol. 2012;13(1):25-32.

19. Schneeweiss A, Chia S, Hickish T, Harvey V, Eniu A, et al. Pertuzumab plus trastuzumab in combination with standard neoadjuvant anthracycline-containing and anthracycline-free chemotherapy regimens in patients with HER2positive early breast cancer: a randomized phase II cardiac safety study (TRYPHAENA). Ann Oncol. 2013;24(9):2278-84.

20. Untch M, Jackisch C, Schneeweiss A, Conrad B, Aktas B, et al. Nab-paclitaxel versus solvent-based paclitaxel in neoadjuvant chemotherapy for early breast cancer (GeparSepto-GBG 69): a randomised, phase 3 trial. Lancet Oncol. 2016;17(3):345-56.

21. Esserman LJ, Berry DA, Cheang MC, Yau C, Perou $\mathrm{CM}$, et al. Chemotherapy response and recurrence-free survival in neoadjuvant breast cancer depends on biomarker profiles: results from the I-SPY 1 TRIAL (CALGB 150007/150012; ACRIN 6657). Breast Cancer Res Treat. 2012;132(3):1049-62.

22. Nahta R, O'Regan RM. Therapeutic implications of estrogen receptor signaling in HER2-positive breast cancers. Breast Cancer Res Treat. 2012; 135(1):39-48.

23. Gluck S, Arteaga CL, Osborne CK. Optimizing chemotherapy-free survival for the ER/HER2positive metastatic breast cancer patient. Clin Cancer Res. 2011;17(17):5559-61.

24. Bhargava R, Dabbs DJ, Beriwal S, Yildiz IA, Badve $\mathrm{P}$, et al. Semiquantitative hormone receptor level influences response to trastuzumab-containing neoadjuvant chemotherapy in HER2-positive breast cancer. Mod Pathol. 2011;24(3):367-74. 